\title{
Multimodal Imaging of a Vascularized Idiopathic Epiretinal Membrane
}

\begin{tabular}{|c|c|}
\hline Journal: & European Journal of Ophthalmology \\
\hline Manuscript ID & EJO-20-0310.R3 \\
\hline Manuscript Type: & Case Report \\
\hline $\begin{array}{r}\text { Date Submitted by the } \\
\text { Author: }\end{array}$ & 10-Nov-2020 \\
\hline Complete List of Authors: & $\begin{array}{l}\text { Anguita, Rodrigo; Moorfields Eye Hospital NHS Foundation Trust, } \\
\text { Vitreoretinal } \\
\text { Khalid, Hagar; Moorfields Eye Hospital NHS Foundation Trust, Medical } \\
\text { Retina; National Institute for Health Research Biomedical Research } \\
\text { Centre at Moorfields Eye Hospital NHS Foundation Trust and UCL } \\
\text { Institute of Ophthalmology; Tanta University Faculty of Medicine } \\
\text { Arpa, Cristina; Moorfields Eye Hospital NHS Foundation Trust, Medical } \\
\text { Retina; Foundation IRCCS Polyclinic San Matteo } \\
\text { Roth, Janice; Moorfields Eye Hospital NHS Foundation Trust, Medical } \\
\text { Retina } \\
\text { Pal, Bishwanath ; Moorfields Eye Hospital NHS Foundation Trust, Medical } \\
\text { Retina } \\
\text { Keane, Pearse; Moorfields Eye Hospital NHS Foundation Trust, Medical } \\
\text { Retina; National Institute for Health Research Biomedical Research } \\
\text { Centre at Moorfields Eye Hospital NHS Foundation Trust and UCL } \\
\text { Institute of Ophthalmology }\end{array}$ \\
\hline Keywords: & $\begin{array}{l}\text { Epiretinal Membrane }<\text { RETINA, Pars Plana Vitrectomy }<\text { VITREOUS / } \\
\text { ENDOPHTHALMITIS, Arterial Occlusive Disease }<\text { RETINA, Macular Hole } \\
<\text { RETINA, PVD }<\text { RETINA, Retinal Detachment }<\text { RETINA, Retina - } \\
\text { Medical Therapies }<\text { RETINA, Techniques of Retinal Examination }< \\
\text { RETINA }\end{array}$ \\
\hline Abstract: & $\begin{array}{l}\text { Purpose: To report a case of vascularized idiopathic epiretinal membrane } \\
\text { including the multimodal imaging. } \\
\text { Methods: Findings on clinical examination, color fundus photography, } \\
\text { spectral-domain optical coherence tomography, optical coherence } \\
\text { tomography angiography and fluorescein angiography. } \\
\text { Results: 63-year-old woman with a previous retinal detachment who } \\
\text { presented with an asymptomatic idiopathic neovascular complex below } \\
\text { an epiretinal membrane. It was assessed with fluorescein angiography, } \\
\text { OCT and OCT-A. The vascularized ERM was already present previously to } \\
\text { the retinal detachment. } \\
\text { Conclusion: Our case report describes an example of idiopathic epiretinal } \\
\text { membrane complicated by neovascularization; we hypothesized } \\
\text { hypertension could be one of the factors in this case, as it may lead to } \\
\text { decreased retinal perfusion and upregulation in vascular endothelial } \\
\text { growth factor (VEGF). Multimodal imaging can help in detecting } \\
\text { neovascularized ERM. }\end{array}$ \\
\hline
\end{tabular}




\section{SCHOLARONE ${ }^{\text {" }}$ Manuscripts}




\section{MULTIMODAL IMAGING OF A VASCULARIZED IDIOPATHIC EPIRETINAL MEMBRANE}

Rodrigo Anguita ${ }^{1}$; Hagar Khalid ${ }^{1-2-3}$; Cristina Arpa ${ }^{1-4}$; Janice Roth ${ }^{1}$, Bishwanath Pal $^{1}$; Pearse A.

Keane ${ }^{1-2}$

${ }^{1}$ Moorfields Eye Hospital NHS Foundation Trust, London, UK.

${ }^{2}$ NIHR Biomedical Research Centre at Moorfields Eye Hospital NHS Foundation Trust and UCL Institute of Ophthalmology, London, UK.

${ }^{3}$ Ophthalmology Department, Faculty of Medicine, Tanta University, Tanta, Egypt

${ }^{4}$ Ophthalmology Department, Fondazione IRCCS Policlinico San Matteo, Pavia, Italy

Correspondence and reprint requests:

Rodrigo Anguita, MD

Email: rodrigoanguita@gmail.com

The authors have neither financial disclosures nor conflicts of interest. 
Abstract:

Purpose: To report a case of vascularized idiopathic epiretinal membrane including the multimodal imaging.

Methods: Findings on clinical examination, color fundus photography, spectral-domain optical coherence tomography, optical coherence tomography angiography and fluorescein angiography. Results: 63-year-old woman with a previous rhegmatogenous retinal detachment (RRD) who presented with an asymptomatic idiopathic neovascular complex below an epiretinal membrane. It was assessed with fluorescein angiography, OCT and OCT-A. The vascularized ERM was already present previously to the retinal detachment.

Conclusion: Our case report describes an example of idiopathic epiretinal membrane complicated by neovascularization; we hypothesized hypertension could be one of the factors in this case, as it may lead to decreased retinal perfusion and upregulation in vascular endothelial growth factor (VEGF). Multimodal imaging can help in detecting neovascularized ERM.

Introduction: Epiretinal membrane $(E R M)$ can be classified as idiopathic or secondary to different causes such as uveitis, vascular retinal diseases, ocular trauma, and retinal detachment $(\mathrm{RD})^{1}$. It can be either asymptomatic or cause metamorphopsia and visual loss. Histopathologically, ERM is characterized by fibrocellular tissue mainly formed of retinal pigment epithelium (RPE), macrophages, glial cells, fibroblast like cells, extracellular matrix components and vascular endothelial cells. Its classification into vascular and avascular ERM depends on the presence of the vascular elements ${ }^{2}$. We are here presenting multimodal imaging of a vascularized idiopathic ERM in an asymptomatic patient. 


\section{Case Description:}

63-year-old woman with past ocular history of left eye (LE) RRD repaired with vitrectomy two years ago and LE cataract operation 17 months ago. She was referred to a medical retina clinic because of incidental neovascularization found in her LE. Her past medical history included hypertension on treatment with Amlodipine and no evidence of diabetes mellitus. On examination, visual acuity was 6/6 in the right eye (RE) and 6/12 in the LE. Dilated retinal examination revealed a flat neovascular complex below an epiretinal membrane (ERM) originating from the first order temporal vein and some cryotherapy scars seen in the periphery (Fig. A and B). On OCT (Fig. C, D) preretinal hyperreflective lesion seen growing into the ERM was observed. Enface OCT-A (fig. E) demonstrated NVE at the level of superficial capillary plexus slab, corresponding to preretinal hyperreflective vessels with posterior shadowing seen on OCTA B scan (Fig. F), scaffolding the back of the ERM, and demonstrating high flow signals suggesting its activity. Fluorescein angiography (FFA) (Fig. G, H, I) showed retinal neovascularization elsewhere (NVE) leaking into the sub-ERM space. Careful review of previous OCT and color fundus pictures performed 3 years before showed the presence of the ERM and NVE, which have been stable during this period of time $(\mathrm{J}-\mathrm{K})$. The diagnosis of idiopathic vascularized ERM was made. A complete workup was performed which ruled out other causes of NVE.

\section{Conclusions:}

In most cases, a vascularized ERM is secondary to proliferative diabetic retinopathy (PDR) or ischemic retinal vein occlusion (RVO). Our case report describes an example of idiopathic epiretinal membrane complicated by neovascularization, with no evident causes such as PDR or RVO. To the best of our knowledge NVE complicating idiopathic ERM have been described in 
only one previous case report by Gueunoun et $\mathrm{al}^{3}$, and a similar condition associated with Terson syndrome was also reported by Toffoli et al ${ }^{4}$. The authors suggested hypertension as a leading cause for the development of vascularized ERM through vascular endothelial growth factor (VEGF) upregulation secondary to decreased retinal vascular perfusion which could be considered also in our case. Hypertension could be the underlying driving force, still its effects would be expected to be more widespread to the retinal vessels. Another differential diagnosis to consider is branch retinal vein occlusion which may cause an epiretinal membrane in $10-15 \%$ and retina neovascularization in around $10 \%$ of cases 5 . In our case the inferior venous branch was noticed to be considerably dilated compared to the superior one, but there were no signs of retinal ischemia in the FFA. While RRD as a complication of BRVO has been previously reported, this is an infrequently complication, retinal breaks are usually adjacent to neovascular tufts upon which vitreous traction is exerted ${ }^{6}$. In our case It has been documented that the patient had multiple areas of lattice and infero-nasal operculated horse shoe tear which was the cause of RRD. Furthermore, review of older scans of our patient showed that vascularized ERM was present prior to retinal surgery and cryotherapy and so retinal detachment does not seem a likely explanation.

Former histological studies described vascular endothelial growth factor to play a role in the growth of new vessels in previously existing fibrovascular membranes with no evidence of VEGF immunoreactivity in avascular membranes as described by Frank et al ${ }^{7}$, in addition a new research described the histopathological findings of patients with diagnosis of macular pucker did not show evidence of VEGF ${ }^{8}$. However, other studies described the presence of VEGF and FGF (fibroblast growth factor) in both vascular and avascular epiretinal membranes ${ }^{1,9,10}$ wherein angiogenic factors were expressed in proliferative diabetic retinopathy and in relatively avascular proliferative vitreoretinopathy membranes and macular pucker as well. This suggests that even avascular 
membranes could develop abnormal vascularization and raises a question regarding the development of neovascularization secondary to angiogenic factors.

In conclusion, multimodal imaging was able to detect neovascular complex in idiopathic epiretinal membrane which could help in the decision of initiating anti-VEGF treatment, always taking into account the possible risk of contraction of the fibrovascular complex, and for follow up of its response to treatment. 


\section{Bibliography:}

1. Tsanou E, Ioachim E, Stefaniotou M, et al. Immunohistochemical study of angiogenesis and proliferative activity in epiretinal membranes. Int $\mathrm{J}$ Clin Pract 2005;59:1157-1161.

2. Hui $\mathrm{YH}$, Goodnight $\mathrm{R}$, Zhang XJ et al. Glial epiretinal membranes and contraction. Immunohistochemical and morphological studies. Arch Ophthalmol 1988; 106: 12805.

3. Gueunoun $\mathrm{S}$, Nazim G, Bruyère $\mathrm{E}$ et al. Abnormal vascular complex membrane imaged by optical coherence tomography. Retin Cases Brief Rep 2011:5-7.

4. Toffoli D, Allaire GS, Barkat F et al. Membrane in a patient with Terson syndrome. Retin Cases Brief Rep 2010:44-46.

5. Hayreh, S., \& Zimmerman, M. (2015). Fundus Changes In Branch Retinal Arteriolar Occlusion. Retina, 35(10), 2060-2066. Doi: 10.1097/Iae.0000000000000585

6. Cohen, G. (1981). Rhegmatogenous Retinal Detachment Secondary To Branch Retinal Vein Occlusion. Retina, 1(3), 186-189. Doi: 10.1097/00006982-198101030-00009

7. Frank R, Amin R, Eliott $\mathrm{D}$ et al. Basic fibroblastic growth factor and vascular endothelial growth factor are present in epiretinal and choroidal neovascular membranes. Am J Ophthalmol 1996; 122: 393-403.

8. Tognetto, D., De Giacinto, C., D'Aloisio, R., Papagno, C., Pastore, M., \& Zweyer, M. (2018). The Combination of Trypan Blue and Brilliant Blue G-Assisted Vitrectomy for Macular Pucker: Histopathological Findings. Ophthalmologica, 239(2-3), 167-175. doi: $10.1159 / 000485986$ 
9. Armstrong D, Augustin AJ, Spengler R. Detection of VEGF and TNF-a in epiretinal membranes of proliferative diabetic retinopathy proliferative vitreoretinopathy and macular pucker. Ophthalmologica 1998; 212: 410-4.

10. Schneeberger SA, Hjelmeland LM, Tucker RP et al. VEGF and FGF-5 are co-localized in vascular and avascular epiretinal membranes. Am J Ophthalmol 1997; 124: 447-54. 


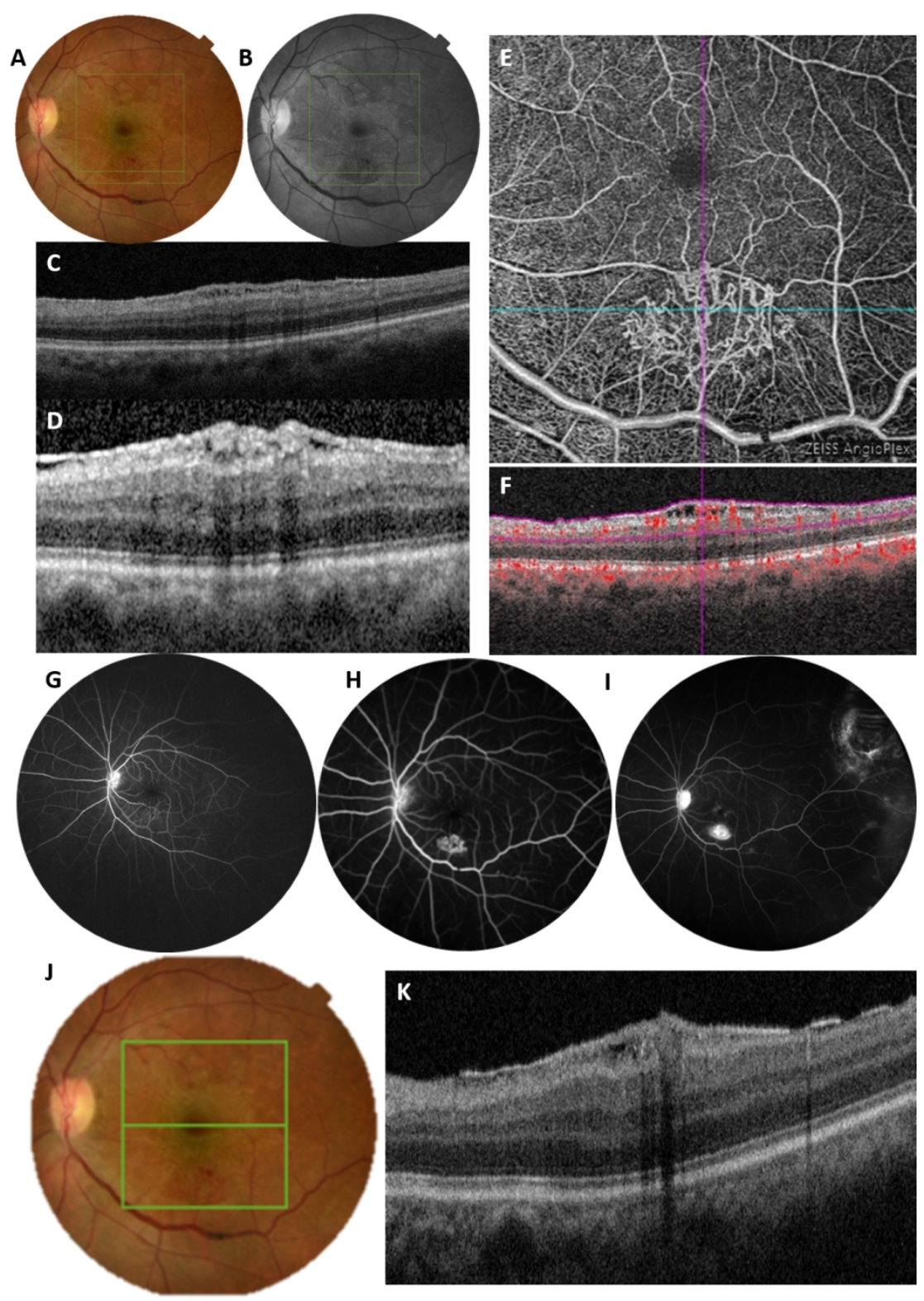

Fig. A (Color fundus picture) and Fig. B (Red-free photograph) flat neovascular complex below an ERM. Fig. $C, D$ (OCT scans) preretinal hyperreflective lesion seen growing into the ERM. Fig. E (Enface OCT -A) and Fig. F (OCT-A B scan) NVE at the level of superficial capillary plexus slab, corresponding to multiple preretinal hyperreflective material seen on OCTA B scan and demonstrating high flow signals suggesting its activity. Fig. G, H, I (Fluorescein angiography) NVE leaking into the sub-ERM space. Fig. J (Color fundus picture) and Fig. K ( OCT scan) Old images showing no changes during the period of follow-up.

$112 \times 156 \mathrm{~mm}(300 \times 300 \mathrm{DPI})$ 